Draft VERSion OCTOBER 24, 2018

Preprint typeset using $\mathrm{LAT}_{\mathrm{E}} \mathrm{X}$ style emulateapj v. 11/10/09

\title{
CONSTRAINING THE EMISSIVITY OF ULTRAHIGH ENERGY COSMIC RAYS IN THE DISTANT UNIVERSE WITH THE DIFFUSE GAMMA-RAY EMISSION
}

\author{
Xiang-Yu Wang ${ }^{1,2,4}$, Ruo-Yu Liu ${ }^{1,4}$, Felix Aharonian ${ }^{3,2}$ \\ Draft version October 24, 2018
}

\begin{abstract}
Ultra-high cosmic rays (UHECRs) with energies $\gtrsim 10^{19} \mathrm{eV}$ emitted at cosmological distances will be attenuated by cosmic microwave and infrared background radiation through photohadronic processes. Lower energy extra-galactic cosmic rays $\left(\sim 10^{18}-10^{19} \mathrm{eV}\right)$ can only travel a linear distance smaller than $\sim \mathrm{Gpc}$ in a Hubble time due to the diffusion if the extra-galactic magnetic fields are as strong as nano Gauss. These prevent us from directly observing most of the UHECRs in the universe, and thus the observed UHECR intensity reflects only the emissivity in the nearby universe within hundreds of Mpc. However, UHECRs in the distant universe, through interactions with the cosmic background photons, produce UHE electrons and gamma-rays that in turn initiate electromagnetic cascades on cosmic background photons. This secondary cascade radiation forms part of the extragalactic diffuse $\mathrm{GeV}-\mathrm{TeV}$ gamma-ray radiation and, unlike the original UHECRs, is observable. Motivated by new measurements of extragalactic diffuse gamma-ray background radiation by Fermi/LAT, we obtained upper limit placed on the UHECR emissivity in the distant universe by requiring that the cascade radiation they produce not exceed the observed levels. By comparison with the gamma-ray emissivity of candidate UHECR sources (such as GRBs and AGNs) at high-redshifts, we find that the obtained upper limit for a flat proton spectrum is $\simeq 10^{1.5}$ times larger than the gamma-ray emissivity in GRBs and $\simeq 10$ times smaller than the gamma-ray emissivity in BL Lac objects. In the case of iron nuclei composition, the derived upper limit of UHECR emissivity is a factor of 3-5 times higher. Robust upper limit on the cosmogenic neutrino flux is further obtained, which is marginally reachable by the Icecube detector and the next-generation detector JEM-EUSO.
\end{abstract}

Subject headings: cosmic rays-gamma-rays: diffuse background-neutrinos

\section{INTRODUCTION}

There is a general consensus that cosmic rays with energy above $10^{19} \mathrm{eV}$ originate from extragalactic astrophysical sources, although the sources are unidentified. Some candidates have been proposed, including active galactic nucleus (AGN) jets (e.g. Biermann \& Strittmatter 1987; Berezinsky et al. 2006), gamma-ray bursts (GRBs) (Waxman 1995; Vietri 1995; Wick et al. 2004; Murase et al. 2008), and semi-relativistic hypernovae remnants (Wang et al. 2007). Any viable candidates must be able to provide a right amount of UHECR emissivity to match the observed flux. Due to the attenuation by cosmic microwave and infrared background radiation through photohadronic processes, the energy loss distance of UHECRs above $10^{19} \mathrm{eV}$ is less than several hundreds of Mpc. Although extragalactic cosmic ray protons in the lower energy range $10^{18} \lesssim E \lesssim 10^{19} \mathrm{eV}$ suffer from much less energy loss, they could possibly travel a linear distance much smaller than the size of the universe as well due to the diffusion in the intergalactic magnetic fields (e.g. Lemoine 2005). For UHECRs whose Larmor radius $r_{L}$ is much larger than the coherence scale $\left(l_{c}\right)$ of the fields,

\footnotetext{
${ }^{1}$ Department of Astronomy, Nanjing University, Nanjing, 210093, China

2 Max-Planck-Institut für Kernphysik, Saupfercheckweg 1, 69117 Heidelberg, Germany

${ }^{3}$ Dublin Institute for Advanced Studies, 31 Fitzwilliam Place, Dublin 2, Ireland

${ }^{4}$ Key laboratory of Modern Astronomy and Astrophysics (Nanjing University), Ministry of Education, Nanjing 210093, China
}

which is valid when $E \gg 10^{18} \mathrm{eV}(B / 1 \mathrm{nG})\left(l_{c} / 1 \mathrm{Mpc}\right)$, the scattering length is $l_{\text {scatt }} \simeq r_{L}^{2} / l_{c}$ and thus the diffusion coefficient5 is $D(E)=(1 / 3) c l_{\text {scatt }}=$ $4 \times 10^{34}\left(E / 10^{18} \mathrm{eV}\right)^{2}(B / 1 \mathrm{nG})^{-2}\left(l_{c} / 1 \mathrm{Mpc}\right) \mathrm{cm}^{2} \mathrm{~s}^{-1}$. In a Hubble time, these particles travel a linear distance $d \sim\left(2 H_{0}^{-1} D\right)^{1 / 2} \simeq$ $60\left(E / 10^{18} \mathrm{eV}\right)(B / 1 \mathrm{nG})^{-1}\left(l_{c} / 1 \mathrm{Mpc}\right)^{-1 / 2} \mathrm{Mpc}, \quad$ where $H_{0}$ is the Hubble constant at present. Therefore, the observed UHECR intensity reflects only the emissivity in the nearby universe within several hundreds of Mpc. Usually, in the literature this emissivity is compared with the emissivity in electromagnetic radiation produced by local candidate sources to check whether they can provide sufficient power. The same type of candidate sources are also present in the distant universe and produce electromagnetic radiation and UHECRs as well. It would be also useful to compare the emissivity of UHECRs and emissivity in electromagnetic radiation in the distant universe, especially when the local sources of the candidates, such as GRBs or AGN giant flares (Farrar \& Gruzinov 2009), are too infrequent to be detectable in years. While the emissivity in the electromagnetic radiation can be measured directly for distant sources, UHECRs produced by them can not be observable and therefore their emissivity can not be determined directly. However, UHECRs produce

5 For extragalactic cosmic ray protons with $E \lesssim$ $10^{18} \mathrm{eV}(B / 1 \mathrm{nG})^{-1}\left(l_{c} / 1 \mathrm{Mpc}\right)$, the smaller angle diffusion approximation will not be valid and the diffusion coefficient in this regime will be different. 
UHE electrons and gamma-rays that in turn initiate electromagnetic cascades on extragalactic background light (EBL) and this lower-energy, secondary cascade radiation can be observable. Thus the energy of UHECRs will be eventually converted to observable diffuse gamma-rays, as was first noticed by Wdowczyk et al. (1972). The spectrum of this cascade radiation is rather insensitive to the spectrum of the original UHECRs (e.g. Strong et al. 1973; Berezinsky \& Smirnov 1975; Coppi \& Aharonian 1997), and thus the total level of the cascade background acts as a particle detector calorimeter, allowing us to measure the total UHECR emissivity in the universe.

Recently, Kalashev et al. (2009) studied the contribution of the cascade radiation by UHECRs interacting with EBL photons to the diffuse extragalactic gammaray background (EGBR) measured by EGRET. Using the new measurement of the EGBR by Fermi/LAT, Berezinsky et al. (2011) and Ahlers et al. (2010) attempted to constrain the UHECR source evolution models and made predictions for the flux level of cosmogenic neutrinos. All these papers assume that the accumulated UHECR flux from a homogenous population of sources in the whole universe fit the observed energy spectrum of UHECRs. In our paper, in order to obtain an independent constraint on the emissivity of UHECRs in the distant universe, we will relax this assumption since UHECRs above $10^{19} \mathrm{eV}$ are produced dominantly by local sources within hundreds of Mpc, much smaller than the size of the universe, whose density could well be enhanced or deficient relative to the average density. Indeed, there are suggestions that one single source in the nearby universe, such as Cen A, is the dominated source producing the observed UHECRs (e.g. Cavallo 1978; Farrar \& Piran 2000; Rieger \& Aharonian 2009).

In $\S 2$, we first present the constraints on the cascade emission imposed by the Fermi/LAT observations of the EGBR. Then in $\S 3.1$, we present an analytic approach for calculating the energy density of the cascade emission produced by UHECRs in the universe. By comparison with the allowed maximum energy density of the cascade radiation measured by Fermi/LAT, we obtain upper limits on the UHECR emissivity at high redshifts in $\S 3.3$. We then discuss the effect of synchrotron loss of secondary electrons in the presence of intergalactic magnetic fields on the cascade radiation in $\S 3.4$. Upper limits on the cosmogenic neutrino flux are further obtained in $\S 3.5$. We discuss the case of heavy nuclei composition of UHECRs in $\S 4$. Finally, we give a summary in $\S 5$.

\section{CASCADE RADIATION}

UHECRs interact with EBL photons through photopion process or Bethe-Heitler process and produce UHE electrons and gamma-rays. These UHE electrons and gamma-rays interact via Compton and pair-production process with soft photons in the CMB and radio background. This will lead to the development of an electromagnetic cascade, in which the number of electrons and photons increase quickly. The cascade proceeds until the energy of photons drops below the pair creation threshold. This process reprocesses essentially all the energy of UHE electrons and gamma-rays produced by UHECRs into lower-energy photons below $\sim \mathrm{TeV}$ which we can detect. The cascade low-energy photon spectrum is a universal spectrum, described by

$$
\frac{d n}{d \epsilon_{\gamma}} \propto\left\{\begin{array}{l}
\epsilon_{\gamma}^{-1.5} \text { for } \epsilon_{\gamma}<\epsilon_{b} \\
\epsilon_{\gamma}^{-\alpha_{\gamma}} \text { for } \epsilon_{b}<\epsilon_{\gamma}<\epsilon_{\text {cut }}
\end{array}\right.
$$

with a steepening at $\epsilon_{\gamma}>\epsilon_{\text {cut }}$, where $\epsilon_{\text {cut }}$ is the absorption energy of a cascade photon scattering on EBL, $\epsilon_{b}=\left(\epsilon_{\text {cut }} / 1 \mathrm{TeV}\right)^{2} \mathrm{GeV}$, and $\alpha_{\gamma} \simeq 1.8-2$ typically (e.g. Berezinsky \& Smirnov 1975; Coppi \& Aharonian 1997). The peak of the energy distribution in this spectrum is at $\epsilon_{\text {cut }}$, which is estimated to be $\sim 100 \mathrm{GeV}$ (Berezinsky et al. 2011). By requiring that this theoretical cascade spectrum touches the lower end of the error bars of the Fermi/LAT data (Abdo et al. 2010), Berezinsky et al. (2011) obtained an upper limit on the cascade energy density, i.e.

$$
\omega_{\gamma} \lesssim \omega_{\text {cas }}^{\max }=5.8 \times 10^{-7} \mathrm{eVcm}^{-3} .
$$

where $\omega_{\gamma}$ is the cascade energy density produced by any UHECR source population in the universe.

\section{CONSTRAINING THE UHECR EMISSIVITY WITH THE} DIFFUSE GAMMA-RAY EMISSION

\subsection{Energy loss of UHECRs into the electro-magnetic component}

Since the cascade emission is mainly in the GeV-TeV range and has an almost universal spectrum, it is numerically much more efficient to calculate the total energy density $\omega_{\gamma}$ injected into the cascade and compare this value with the limit $\omega_{\text {cas }}^{\max }$ imposed by Fermi/LAT. The cascade energy density is the accumulation of the electro-magnetic (EM) radiation produced by the source population over the whole universe. One can calculate it by summing the contributions by individual sources that are generated at different cosmological epochs, i.e.

$$
\omega_{\gamma}=\iint L_{p}(t) \beta_{0, e m}\left(E_{p}^{s}, z(t)\right) d t d E_{p}^{s}
$$

where

$$
L_{p}(t) \equiv \dot{n}(z) E_{p}^{s} \frac{d N_{p}}{d E_{p}^{s}},
$$

is the emissivity of protons per energy decade at energy $E_{p}^{s}$ at some cosmological epoch $t, \beta_{0, e m}$ is the fraction of the proton energy deposited into the EM component (photons and pairs) that we observed at present, $\dot{n}(z)$ is the comoving-frame number density of protons injected per unit time at time $t=t(z), E_{p}^{s}$ is the proton energy at the source, and $\frac{d N_{p}}{d E_{p}^{s}}$ is the energy spectrum of protons.

The energy of injected protons evolves with time as

$$
-\frac{d E_{p}}{d t}=E_{p} H(z)+b\left(E_{p}, t\right)
$$

where $H(z)$ is the Hubble constant at time $t=t(z)$, and

$$
\begin{aligned}
& b\left(E_{p}, t\right)=\frac{E_{p} c}{2 \Gamma^{2}} \int_{\varepsilon_{t h}}^{\infty} d \varepsilon_{\gamma}\left[\sigma_{\mathrm{BH}}\left(\varepsilon_{\gamma}\right) f_{\mathrm{BH}}\left(\varepsilon_{\gamma}\right)+\sigma_{p \gamma}\left(\varepsilon_{\gamma}\right) f_{p \gamma}\left(\varepsilon_{\gamma}\right)\right] \\
& \times \varepsilon_{\gamma} \int_{\varepsilon_{\gamma} / 2 \Gamma}^{\infty} d \varepsilon \frac{n_{\gamma}(\varepsilon, z)}{\varepsilon^{2}}
\end{aligned}
$$

is the total energy loss rate of protons of energy $E_{p}$ due to photopair and photopion interactions with EBL photons at some cosmological time $t(z), \Gamma$ is the proton Lorentz factor, $\sigma_{\mathrm{BH}}$ and $\sigma_{p \gamma}$ are the cross section for photopair 
and photopion production respectively, $f_{\mathrm{BH}}$ and $f_{p \gamma}$ are the fractions of energy loss of protons due to photopair and photopion interactions in one collision, and $n_{\gamma}(\varepsilon, z)$ is the number density of EBL photons of energy $\varepsilon$ at redshift $z$. We use the cross section in Chodorowski et al. (1992) for the Bethe-Heitler process and use the full photopion production cross section from the pion production threshold up to high energies as described in Mücke et al. (2000). The EBL includes CMB photons and infrared-to-optical background photons (Finke et al. 2010). The number density of infrared-to-optical background photons at high-redshifts are taken from the data set online 6 .

From the above equations, one can solve $E_{p}(t)$. Then one can obtain the energy loss of protons exclusively into the EM component per unit time at some cosmological time $t(z)$,

$$
\begin{aligned}
& \frac{d E_{p, e m}(z)}{d t}=\frac{E_{p} c}{2 \Gamma^{2}} \int_{\varepsilon_{t h}}^{\infty} d \varepsilon_{\gamma}\left[\sigma_{\mathrm{BH}}\left(\varepsilon_{\gamma}\right) f_{\mathrm{BH}}\left(\varepsilon_{\gamma}\right)\right. \\
& \left.+R_{\mathrm{em}} \sigma_{p \gamma}\left(\varepsilon_{\gamma}\right) f_{p \gamma}\left(\varepsilon_{\gamma}\right)\right] \times \varepsilon_{\gamma} \int_{\varepsilon_{\gamma} / 2 \Gamma}^{\infty} d \varepsilon \frac{n_{\gamma}(\varepsilon, z)}{\varepsilon^{2}}
\end{aligned}
$$

where $R_{e m} \simeq 0.6$ is the fraction of proton energy that goes into the EM component in the photopion channel (the other $\simeq 0.4$ goes into the neutrino production channel) (Engel et al. 2001).

Then the energy lost into the EM component by a proton of energy $E_{p}^{s}$ (at the source) during the whole period from the injection time $t(z)$ to the present time is

$\beta_{e m}\left(E_{p}^{s}, t\right) E_{p}^{s} \equiv \int_{0}^{t(z)} \frac{d E_{p, e m}(z)}{d t} d t=\int_{0}^{z} \frac{d E_{p, e m}(z)}{d t} \frac{d t}{d z} d z$.

The corresponding energy that remains at the present epoch after taking into account the redshift energy loss is

$$
\beta_{0, e m}\left(E_{p}^{s}, t\right) E_{p}^{s} \equiv \int_{0}^{t(z)} \frac{1}{1+z} \frac{d E_{p, e m}(z)}{d t} d t .
$$

The values of $\beta_{e m}\left(E_{p}^{s}, t\right)$ as a function of the energy of protons generated at different redshifts are shown in Fig.1. For a source at $z \gtrsim 0.5$, more than a half of the proton energy is lost into the EM cascade for protons with energy above $10^{18.5} \mathrm{eV}$.

\subsection{The UHECR source density evolution}

The evolution of the UHECR emissivity per energy decade with redshift can be parameterized as

$$
L_{p}(z)=L_{p}(z=1) \frac{S(z)}{S(z=1)}
$$

where $L_{p}(z=1) \equiv \dot{n}(z=1) E_{p}^{s} \frac{d N_{p}}{d E_{p}^{s}}, \dot{n}(z=1)$ is the comoving-frame number density of protons injected per unit time at $z=1$ and $S(z)$ is the source density at redshift $z$. We take three cases for $S(z)$ in the following calculation, i.e. $S(z)$ follows the star formation history (SFR), gamma-ray burst (GRB) rate and active galactic nuclei (AGN) rate in the universe respectively.

We take the form of SFR from Yüskel et al. (2008)

$$
S_{\mathrm{SFH}}(z) \propto\left\{\begin{array}{l}
(1+z)^{3.4}, \quad z<1 \\
(1+z)^{-0.3}, \quad 1<z<4 \\
(1+z)^{-3.5}, \quad z>4
\end{array}\right.
$$

Recent analysis of the GRB redshift distribution as detected by Swift reveals that the GRB rate is enhanced at high redshift relative to SFR (Le \& Dermer 2007). This may arise from some mechanisms (Kistler et al. 2008), such as a GRB preference for low-metallicity environments (Stanek et al. 2006; Langer \& Norman 2006). Following Yuksel \& Kistler (2007), we assume $S_{\mathrm{GRB}}(z) \propto(1+z)^{1.4} S_{\mathrm{SFH}}$, which gives

$$
S_{\mathrm{GRB}}(z) \propto\left\{\begin{array}{l}
(1+z)^{4.8}, \quad z<1 \\
(1+z)^{1.1}, \quad 1<z<4 \\
(1+z)^{-2.1} . \quad z>4
\end{array}\right.
$$

As shown by Yuksel \& Kistler (2007), this source density evolution function is consistent with the evolution function obtained in Le \& Dermer (2007).

AGNs may have a similarly strong evolution with redshift, as found in Hasinger et al. (2005) for different luminosity AGNs. Following Hasinger et al. (2005) and Ahlers et al. (2009), we take the form of

$$
S_{\mathrm{AGN}}(z) \propto\left\{\begin{array}{l}
(1+z)^{5.0}, \quad z<1.7 \\
\text { constant, } 1.7<z<2.7 \\
10^{(2.7-z)}, \quad z>2.7
\end{array}\right.
$$

\subsection{Constraints on the emissivity of UHECRs in the distant universe}

Once we know $\beta_{0, e m}$ and the source density evolution function $S(z)$, we can calculate the cascade energy density $\omega_{\gamma}$, i.e.

$\omega_{\gamma}=\int_{0}^{z_{\max }} \int_{E_{p, \min }}^{E_{p, \max }} \beta_{0, e m}\left(E_{p}^{s}, z\right) L_{p}(z=1) \frac{S(z)}{S(z=1)} \frac{d t}{d z} d E_{p}^{s} d z$,

where $E_{p, \min }$ and $E_{p, \max }$ are the minimum and maximum energy of extragalactic cosmic rays, $z_{\max }$ is the maximum redshift of extragalactic cosmic ray sources, and $d z / d t=$ $H_{0}(1+z)\left[\Omega_{M}(1+z)^{3}+\Omega_{\Lambda}\right]^{1 / 2}\left(\right.$ with $\Omega=0.3, \Omega_{\Lambda}=0.7$, and $\left.H_{0}=70 \mathrm{kms}^{-1} \mathrm{Mpc}^{-1}\right)$. We assume a power-law spectrum for extragalactic cosmic rays between $E_{p \text {,min }}$ and $E_{p, \max }$, i.e. $d N_{p} / d E_{p}^{s} \propto\left(E_{p}^{s}\right)^{-\gamma_{g}}$. As $\beta_{e m}$ becomes negligible at $E_{p}<10^{17} \mathrm{eV}, E_{p, \min }$ is taken to be $10^{17} \mathrm{eV}$ in the following calculations, unless otherwise specified.

Requiring $\omega_{\gamma} \lesssim \omega_{\text {cas }}^{\max }$, one can obtain the upper limit on the UHECR emissivity at $z=1$ for different source density evolution scenarios. We define the total UHECR emissivity as the integral of $L_{p}(z=1)$ over the energy range from $E_{p, \min }$ to $E_{p, \max }$, i.e.

$$
P(z=1) \equiv \int_{E_{p, \min }}^{E_{p, \max }} L_{p}(z=1) d E_{p}^{s}
$$

The upper limits of $P(z=1)$ for different spectral in$\operatorname{dex} \gamma_{g}$ are shown in Fig.2 (the upper panel) with a fixed maximum proton energy $E_{p, \max }=10^{21} \mathrm{eV}$ (but different minimum energies) for different source density evolution scenarios. The values are in the range of a few $10^{45}$ to a few $10^{46} \mathrm{ergMpc}^{-3} \mathrm{yr}^{-1}$. The upper limit for the SFH case is the highest because of the slowest redshift evolution and smaller $\beta_{0, e m}$ at lower redshifts. As a comparison, we also show the gamma-ray emissivity of GRBs and BL Lac objects at redshift $z=1$. Though 
the local gamma-ray emissivity of GRBs inferred by different groups are different by about one order of magnitude (Guetta et al. 2005; Le \& Dermer 2007; Wanderman \& Piran 2010), the inferred emissivity in GRBs at redshift $z=1$ has a much smaller uncertainty, being $1.1 \times 10^{44}(\Delta t / 10 \mathrm{~s}) \mathrm{ergMpc}^{-3} \mathrm{yr}^{-1}$ in Guetta et al. $(2005), 1.8 \times 10^{44}(\Delta t / 10 \mathrm{~s}) \mathrm{ergMpc}^{-3} \mathrm{yr}^{-1}$ in Wanderman \& Piran (2010), and $2.5 \times 10^{44}(\Delta t / 10 \mathrm{~s}) \operatorname{ergMpc}^{-3} \mathrm{yr}^{-1}$ in Le \& Dermer (2007), where $\Delta t$ is the mean duration of long GRB in the explosion frame. By comparison with the upper limits on the UHECR emissivity, we find that the ratio between the two emissivities is $P_{\mathrm{CR}}\left(10^{17}-10^{21} \mathrm{eV}\right) / P_{\gamma} \lesssim 10^{1.5}$ for a flat cosmic ray spectrum $\gamma_{g} \simeq 2.0$.

Dermer \& Razzaque (2010) recently obtained the gamma-ray emissivity of different classes of AGNs. The two bright classes at high redshift are BL Lac objects and flat spectrum radio quasars (FSRQs). The gamma-ray emissivity in BL Lac objects and FSRQs at $z=1$ are, respectively, $6 \times 10^{46} \mathrm{ergMpc}^{-3} \mathrm{yr}^{-1}$ and $1.5 \times 10^{46} \mathrm{ergMpc}^{-3}$. If these sources produce UHECRs, we have $P_{\mathrm{CR}}\left(10^{17}-10^{21} \mathrm{eV}\right) / P_{\gamma} \lesssim 0.1$ for $\gamma_{g} \simeq 2.0$. This suggests that either a small fraction of the BL Lac objects in the distant universe are capable of accelerating protons to ultra-high energies or the baryon-electron ratio in these sources is significantly smaller than one.

Though there are few astrophysical sources that can accelerate protons to energies beyond $10^{20} \mathrm{eV}$, there should be more sources in the universe that can accelerate protons to a lower $E_{p, \max }$. For example, accretion shocks in clusters of galaxies may be able to accelerate protons to $10^{18}-10^{19} \mathrm{eV}$ (e.g. Inoue et al. 2005). These ultrahigh energy protons produce diffuse gamma-ray emission as well. Therefore, the observed extragalactic diffuse gamma-ray background also constrains the UHECR emissivity produced by these lower $E_{p, \max }$ accelerators. The upper limits of UHECR emissivity for different $E_{p, \max }$ are shown in Fig.3. It shows that for lower $E_{p, \max }$ accelerators, the UHECR emissivity could be higher, but it must still be lower than $\sim 10^{47} \mathrm{ergMpc}^{-3} \mathrm{yr}^{-1}$ at $z=1$.

\subsection{The effect of intergalactic magnetic field on the cascade radiation}

When the intergalactic magnetic fields are strong, the synchrotron cooling of the secondary electrons produced by UHECRs propagating in the intergalactic space could dominate over the inverse-Compton cooling and as a result, the cascade energy is reduced to some extent compared to the case without the magnetic fields. Unfortunately, very little is known about the origin, spatial configuration and amplitude of the intergalactic magnetic fields. Only upper limit on the intergalactic magnetic fields is obtained from Faraday rotation measurements, i.e. $B \lesssim 10^{-8} \mathrm{G}\left(l_{c} / 1 \mathrm{Mpc}\right)^{-1 / 2}$, where $l_{c}$ is the coherence length scale of the fields (e.g. Ryu et al. 1998). Following Gabici \& Aharonian (2005) and Kotera et al. (2011), the effective inverse Compton cooling time on the $\mathrm{CMB}$ and radio backgrounds can be written as $t_{e \gamma} \simeq 5 \times 10^{14} \mathrm{~s}\left(E_{e} / 10^{18} \mathrm{eV}\right)^{\alpha_{I C}}$, with $\alpha_{I C}=1$ if the electron energy $E_{e} \lesssim 10^{18} \mathrm{eV}$ and $\alpha_{I C}=0.25$ if $10^{18} \mathrm{eV} \lesssim E_{e} \lesssim 10^{20} \mathrm{eV}$. $\widetilde{\text { Above }} 10^{20} \mathrm{eV}, t_{e \gamma} \simeq 1.6 \times$ $10^{15} \mathrm{~s}\left(E_{e} / 10^{20} \mathrm{eV}\right)$. In comparison, the synchrotron cooling time is $t_{e B} \simeq 4 \times 10^{14} \mathrm{~s}(B / 1 \mathrm{nG})^{-2}\left(E_{e} / 10^{18} \mathrm{eV}\right)^{-1}$.
The opposite scalings of $t_{e \gamma}$ and $t_{e B}$ with electron energy imply the existence of a cross-over energy $E_{e, c r}$, above which electrons cool mainly via synchrotron radiation instead of undergoing an inverse Compton cascade, i.e. $E_{e, c r}=10^{18} \mathrm{eV} B_{\mathrm{nG}}^{-1}$ for $B \gtrsim 1 \mathrm{nG}, E_{e, c r}=10^{18} \mathrm{eV} B_{\mathrm{nG}}^{-1.6}$ for $1 \mathrm{nG} \gtrsim B \gtrsim 0.1 \mathrm{nG}$, and $E_{e, c r}=5 \times 10^{20} \mathrm{eV} B_{0.01 \mathrm{nG}}^{-1}$ for $B \lesssim 0.1 \mathrm{nG}$ (Kotera et al. 2011). Since the energy of secondary electrons produced by UHE protons is a fraction of $1 / 20$ or $10^{-3}$ of the parent proton energy in the photopion or photopair process, electrons with energies above $E_{e, c r} \gtrsim 10^{18} \mathrm{eV}$ can be produced only by the photopion process of UHE protons. In Fig.4, we show the fraction energy loss of UHE protons into the cascade EM component (i.e. excluding the synchrotron radiation) in the presence of the intergalactic magnetic fields (for three cases of $B=0.01,0.1$ and $1 \mathrm{nG}$ ). It shows that this fraction drops significantly as the magnetic fields increase for protons above the photopion threshold energy. We further calculate the cascade energy density $\omega_{\gamma}^{B}$ in the presence of intergalactic magnetic fields. In Fig.5, we show the ratio of the cascade energy densities in the presence of a magnetic field $\left(\omega_{\gamma}^{B}\right)$ and in its absence $\left(\omega_{\gamma}\right)$ as a function of the maximum proton energy for different UHECR source populations. When $E_{p, \max }$ is large, up to $\sim 45 \%$ of the cascade energy is lost into the synchrotron radiation.

The characteristic energy of the synchrotron radiation is $E_{\gamma, \text { syn }} \simeq 6.8 \mathrm{GeV} B_{n G}\left(E_{e} / 10^{19} \mathrm{eV}\right)^{2}$. So when the magnetic fields are stronger, e.g. $1 \mathrm{nG}<B<10 \mathrm{nG}$, the synchrotron peak falls at $10-100 \mathrm{GeV}$. Thus the synchrotron component in fact also contributes to EGRB, although its spectrum is different from that of the cascade radiation. Therefore we conclude that the presence of intergalactic magnetic fields affects the upper limits of the UHECR emissivity by a factor $\lesssim 2$.

\subsection{The upper bound on the cosmogenic neutrino flux}

Once we know the upper bound on the UHECR emissivity, we can get the upper bound on the cosmogenic neutrino flux. As the energy loss distance for protons of energy above the photopion production threshold in interactions with $\mathrm{CMB}$ photons is relatively short $(\lesssim 300$ $\mathrm{Mpc}$ ), it is reasonable to assume that cosmic rays at these energies lose all of their energy locally. Following Engel et al. (2001) and Yuksel \& Kistler (2007), we use the approximation that the fraction of the original proton energy that is lost to neutrinos can be parametrized with a gradual step function $\psi\left(E_{p}\right)=0.45 /\left(1+\left(E_{t} / E_{p}\right)^{2}\right)$, where 0.45 is the asymptotic fraction of injected cosmicray energy transferred to neutrinos above $10^{21} \mathrm{eV}$ and $E_{t} \sim 2 \times 10^{20} \mathrm{eV}$. Thus, the total $\nu_{\mu}+\bar{\nu}_{\mu}$ energy flux at Earth can be written as a sum of the contributions by individual UHECR sources that are generated at different cosmological epochs, i.e.

$$
E_{\nu} J_{\nu}=\frac{c}{4 \pi} \int_{0}^{z_{\max }} \frac{1}{1+z} \psi\left(E_{p}^{s}(1+z)\right) L_{p}(t) \frac{d E_{p}^{s}}{d E_{\nu}} \frac{d t}{d z} d z
$$

where $E_{p}^{s}=20(1+z) E_{\nu}$ (approximating each daughter neutrino receiving about $1 / 20$ of the injected proton energy).

The results are shown in Fig.6 for $E_{p, \min }=10^{17} \mathrm{eV}$, $E_{\max }=10^{21} \mathrm{eV}$ and a flat proton spectrum with $\gamma_{g}=2$. 
In contrast to Berezinsky et al. (2011) and Ahlers et al. (2010), we relax the requirement that the accumulated UHECR flux from a homogenous population of sources in the whole universe fit the observed energy spectrum of UHECRs since UHECRs above $10^{19} \mathrm{eV}$ are produced dominantly by local sources within hundreds of $\mathrm{Mpc}$ and the UHECR emissivity in the distant universe could be irrelevant. As a result, the upper bound on cosmogenic neutrino flux shown in Fig.6 represents a true upper bound that is independent of the unknown density distribution of nearby sources that contribute to the observed UHECR flux. This upper bound is below the sensitivity of Auger and is marginally reachable by Icecube and the next-generation detector JEM-EUSO.

The commonly-used Waxman-Bahcall bound (Waxman \& Bahcall 1999) for high-energy neutrinos also assumes that UHECR emissivity in the distant universe is connected with the flux of observed UHECRs that are produced by local sources. Without any independent constraints, the UHECR emissivity in the distant universe could in principal be arbitrarily high. However, with the upper limit of the cascade radiation, the constraints on the UHECR emissivity in the distant universe becomes possible now and hence the neutrino upper bound becomes solid.

\section{THE IRON NUCLEI CASE}

The composition of UHECRs remains disputed. Although HiRes observations favor proton composition (Abbasi et al. 2010), recent observations by Pierre Auger Observatory (PAO) show a transition in the maximum shower elongations $\left\langle\mathrm{X}_{\max }\right\rangle$ and in their fluctuations $\operatorname{RMS}\left(\mathrm{X}_{\max }\right)$ between $5 \mathrm{EeV}$ and $10 \mathrm{EeV}$ (Abraham et al. 2010), which are interpreted as reflecting a transition in the composition of UHECR in this energy range from protons to heavier mass nuclei. However, one should be cautious that this claim depends on the poorly-understood hadronic interaction models at such high energies. In this section, we study the heavy nuclei composition case. For simplicity, a pure iron composition above $10^{19} \mathrm{eV}$ is assumed in our calculation and the maximum energy of iron nuclei is fixed at $10^{21} \mathrm{eV}$. As the corresponding maximum energy of one nucleon is only $1.8 \times 10^{19} \mathrm{eV}$, which is below the threshold energy for photopion production of protons interacting with CMB photons even at high redshifts, we can neglect the photopion energy loss for these UHE iron nuclei.

For an Fe nucleus generated at cosmological time $t(z)$, it suffers from both energy loss due to Bethe-Heitler process and nucleon loss due to photo-disintegration during the propagation in the intergalactic space. Let's denote $\gamma_{N}(t)$ as the Lorentz factor of the nucleus and $A(t)$ as the mass number of the nucleus. As the Lorentz factor of the nucleus is conserved during photo-disintegration, the energy loss is due to Bethe-Heitler cooling and adiabatic expansion of the universe. The energy loss due to de-excitation of nuclei following photo-disintegration interactions is found to be always less efficient than the Bethe-Heitler energy loss (Aharonian \& Taylor 2010), so it can be safely neglected. So the the evolution of $\gamma_{N}(t)$ with time is given by

$$
\frac{d \gamma_{N}(t)}{d t}=\gamma_{N}(t) H(z)+\dot{\gamma}_{N, B H}(t, A),
$$

where

$$
\begin{aligned}
& \dot{\gamma}_{N, B H}(t, A)=\frac{Z^{2}}{A} \dot{\gamma}_{p, B H}(t) \\
& =\frac{Z^{2}}{A} \frac{c}{2 \gamma_{N}} \int_{\varepsilon_{t h}}^{\infty} d \varepsilon_{\gamma} \sigma_{B H}\left(\varepsilon_{\gamma}\right) f\left(\varepsilon_{\gamma}\right) \varepsilon_{\gamma} \int_{\varepsilon_{\gamma} / 2 \Gamma}^{\infty} d \varepsilon \frac{n_{\gamma}(\varepsilon)}{\varepsilon^{2}}
\end{aligned}
$$

is the Bethe-Heitler energy loss rate for nucleus of charge $Z$ and mass number $A, \dot{\gamma}_{p, B H}(t)$ is the Bethe-Heitler energy loss rate for protons of the same Lorentz factor $\gamma_{N}$. The photo-disintegration results in nucleon loss of nuclei, so the mass number evolves with time as

$$
\frac{d A(t)}{d t}=R_{A}\left(t, \gamma_{N}\right)
$$

where

$$
R_{A}\left(t, \gamma_{N}\right)=\frac{c}{2 \gamma_{N}^{2}} \int_{\varepsilon_{\mathrm{th}}}^{\infty} d \varepsilon \sigma_{A}(\varepsilon) \varepsilon \int_{\varepsilon / 2 \gamma_{N}}^{\infty} d x x^{-2} n_{\gamma}(x)
$$

is the total photo-disintegration rate for nucleus with Lorentz factor $\gamma_{N}$ and mass number $A, \sigma_{A}(\epsilon)$ is the total photodisintegration cross section, and $\varepsilon_{\mathrm{th}}$ is the threshold energy of the photon in the nucleus rest frame.

It is found that the single nucleon loss is the dominant channel for photo-disintegration of heavy nuclei (Puget et al. 1976), so as a good approximation, we here only consider single nucleon loss channel in the following calculation. This approximation results in an error less than $30 \%$ for nucleus with Lorentz factor smaller than $2 \times 10^{10}$ (corresponding to energy $\lesssim 10^{21} \mathrm{eV}$ for an iron nucleus)(Puget et al. 1976). The cross-sections for photodisintegration in the energy range $\varepsilon_{t h}<\varepsilon \lesssim 30 \mathrm{MeV}$ with single nucleon loss is dominated by the giant dipole resonance (GDR), which can be approximately described by a Lorentzian form (Puget et al. 1976; Anchordoqui et al. 2007) as

$$
\sigma_{A}(\varepsilon)=\frac{\sigma_{0, A} \varepsilon^{2} \Delta_{G D R}^{2}}{\left(\varepsilon_{0}^{2}-\varepsilon^{2}\right)^{2}+\varepsilon^{2} \Delta_{G D R}^{2}},
$$

where $\Delta_{G D R}$ and $\sigma_{0, A}$ are the width and maximum value of the cross section, $\varepsilon_{0}$ is the energy at which the cross section peaks. Fitted numerical values are $\sigma_{0, A}=1.45 A \times 10^{-27} \mathrm{~cm}^{2}, \Delta_{G D R}=8 \mathrm{MeV}$, and $\varepsilon_{0}=42.65 A^{-0.21}\left(0.925 A^{2.433}\right) \mathrm{MeV}$ for $A>4(A<4)$ (Karakula \& Tkaczyk 1993).

Combining Eq.(18) and Eq.(20), one can obtain $\gamma_{N}(t)$ and $A(t)$. Denote $t_{A=1}$ as the time when the mass number of a parent nucleus drops to $A=1$. Then, one can calculate the energy lost into the EM component by an Fe nucleus with an initial energy $E_{N}^{s}$ during the whole period from the the injection time $t(z)$ to the present time

$$
\begin{aligned}
& \beta_{e m}^{N}\left(E_{N}^{s}, t\right) E_{N}^{s} \equiv \int_{t_{A=1}}^{t(z)}\left[\dot{\gamma}_{N, B H}(t, A) A(t)+(56-A)\right. \\
& \left.\times \dot{\gamma}_{p, B H}(t)\right] m_{p} c^{2} d t+\int_{0}^{t_{A=1}}\left[56 \dot{\gamma}_{p, B H}(t)\right] m_{p} c^{2} d t,
\end{aligned}
$$

where the second term on the right side is the BetheHeitler energy loss for those secondary nucleons that have been already disintegrated from the parent nucleus. Note that, for the sake of analytic calculations, we have assumed that all the secondary nucleons that have been disintegrated from the parent nucleus have an averaged $\gamma_{p}$ at some time $t$, which is a good approximation since 
the photo-disintegration process of nuclei in the majority of the relevant energy range is faster than the BetheHeitler cooling (Puget et al. 1976; Stecker \& Salamon 1999; Ave et al. 2005) 7 . In Fig.7, we show $\beta_{e m}^{N}$ as a function of the energy of iron nucleus generated at different redshifts. Note that the bumps in these curves correspond to the energies of those iron nuclei that interact with CMB photons at the GDR peak. The rise of $\beta_{e m}^{N}$ at higher energies is due to the contributions by the nucleons that have been disintegrated from the parent nucleus. For an iron nucleus generated at $z \gtrsim 1$ with an energy $\gtrsim 5 \times 10^{19} \mathrm{eV}$, more than a half of the nucleus energy is lost into the EM cascade radiation. Compared with the proton case, the energy loss fraction is smaller at energies $E_{\mathrm{Fe}} \lesssim 10^{20} \mathrm{eV}$.

Similar to the proton case, we can calculate the corresponding energy that remains at the present epoch, $\beta_{0, e m}^{N} E_{N}^{s}$, by taking into account the redshift energy loss. Finally, we obtain the cascade energy density produced by the whole UHECR source population in the universe

$\omega_{\gamma, N}=\int_{0}^{z_{\max }} \int_{E_{N, \min }}^{E_{N, \max }} \beta_{0, e m}^{N}\left(E_{N}^{s}, z(t)\right) L_{N}(z=1) \frac{S(z)}{S(z=1)} \frac{d t}{d z}$

where $L_{N}(z=1)$ is the emissivity of UHE iron nuclei per energy decade at $z=1$. Requiring $\omega_{\gamma, N} \lesssim \omega_{\text {cas }}^{\max }$, we obtain the upper limits on the UHECR emissivity at redshift $z=1$ (i.e. $P(z=1)$ ) for different source evolution scenarios, which are shown in Fig.8. Note that for the iron composition case, $P(z=1)$ is defined as the integral of $L_{N}(z=1)$ over the energy range from $10^{19} \mathrm{eV}$ to $10^{21} \mathrm{eV}$. These upper limits are a factor of 3-5 times higher than that in the proton composition case due to smaller energy loss fractions $\beta_{0, \text { em }}^{N}$ at energies $E_{\mathrm{Fe}} \lesssim 10^{20}$ $\mathrm{eV}$.

\section{SUMMARY AND DISCUSSIONS}

The observed UHECR intensity above $10^{19} \mathrm{eV}$ reflects only the UHECR emissivity in the nearby universe due to that these extremely high-energy particles lose energy quickly while propagating in the universe. Thus, there is no direct information about the UHECR emissivity in the distant universe. In this paper, we suggest that the cascade gamma-ray radiation initiated by the secondary particles produced by UHECRs provides a useful probe of the UHECR emissivity in the distant universe, since this cascade gamma-ray radiation can be observed directly. Using the new Fermi/LAT measurement of the EGBR, we obtained upper limits on the UHECR emissivity in the distant universe through an analytic treatment. Both proton composition and pure iron composition cases are studied. These limits are then compared with the gamma-ray emissivity of candidate UHECR sources, i.e. GRBs and AGNs, at high redshifts. We find that, for a flat proton spectrum, the upper limit of the UHECR emissivity is a few tens times larger than the observed gamma-ray emissivity in GRBs, while it is about one order of magnitude smaller than the gamma-ray emissivity in BL Lac objects. We also find that the presence of intergalactic magnetic fields has insignificant effect on the derived upper limit. Furthermore, an upper limit on the cosmogenic neutrino flux is obtained from the upper limit of UHECR emissivity. In contrast to Berezinsky et al. (2011) and Ahlers et al. (2011), we relax the assumption that the accumulated UHECR flux from a homogenous population of sources in the whole universe fit the observed UHECR energy $d t$ spectyum, since UHECRs above $10^{19} \mathrm{eV}$ originate from zlocal sources within hundreds of Mpc and the UHECR emissivity in the distant universe could be irrelevant. Therefore our upper limit on the cosmogenic neutrino flux represents a true upper bound that is independent of the unknown density distribution of the nearby sources that contribute to the observed UHECR flux. This upper bound is below the sensitivity of Auger and is only marginally reachable by Icecube and the future detector JEM-EUSO.

Recently, Neronov \& Semikoz (2011) obtained the flux of the EGBR using a different method from that used in Abdo et al. (2010), which is a factor of $\simeq 2$ below the flux obtained in Abdo et al. (2010) in the 100-200 $\mathrm{GeV}$ range. If this flux is true, the upper limits on the UHECR emissivity as well as the upper bounds on the cosmogenic neutrinos will go down by the same factor of $\simeq 2$.

We thank the referee for the helpful comments and suggestions. This work is supported by the NSFC under grants 10973008 and 11033002 , the 973 program under grant 2009CB824800, the Program for New Century Excellent Talents in University, the Qing Lan Project and the Fok Ying Tung Education Foundation.

\section{REFERENCES}

Abbasi, R. U. et al. 2010, Phys. Rev. Lett., 104, 161101

Abdo, A. A., et al., 2010, Phys. Rev. Lett., 104, 101101

Abraham, J. et al. 2010, Phys. Rev. Lett., 104, 091101

Aharonian, F. \& Taylor, A. M., 2010, Astroparticle Physics 34, 258

Ahlers, M.; Anchordoqui, L. A.; Sarkar, S., 2009, Phys. Rev. D, 79, 083009

Ahlers, M.; Anchordoqui, L. A.; GonzalezCGarcia, M. C.; Halzen, F.; Sarkar, S., 2010, Astroparticle Physics, 34, 106

\footnotetext{
7 In the small energy range where the Bethe-Heitler cooling is more efficient (e.g. Ave et al. 2005), the energy loss of parent nucleus into the EM cascade dominate over the energy loss by the disintegrated nucleons since the Bethe-Heitler cooling efficiency scales as $Z^{2} / A$, therefore Eq.(22) remains a good approximation.
}

Anchordoqui, L. A.; Beacom, J. F.; Goldberg, H.; Palomares-Ruiz, Se.; Weiler, T. J., 2007, Phys. Rev. D, 75, 063001

Ave, M.; Busca, N.; Olinto, A. V.; Watson, A. A.; Yamamoto, T., 2005, Astroparticle Physics, 23, 19

Biermann, P. L., \& Strittmatter, P. A. 1987, ApJ, 322, 643

Berezinsky, V., A. Yu. Smirnov, 1975, Astrophys. Sp. Sci. 32,461

Berezinsky, V., Gazizov, A., \& Grigorieva, S., 2006, Phys. Rev. D, 74,043005

Berezinsky, V.; Gazizov, A.; Kachelrieß, M.; Ostapchenko, S., 2011, Physics Letters B, 695, 13

Cavallo, G, 1978, A\&A, 65, 415

Chodorowski, M. J., Zdziarski, A. A., \& Sikora, M. 1992, ApJ, 400,181

Coppi, P. S. \& Aharonian, F. A., 1997, ApJ, 487, L9

Dermer. C. D. \& Razzaque, S., 2010, ApJ, 724, 1366

Engel, R., Seckel, D. \& Stanev, T., 2001, Phys. Rev. D, 64, 093010 
Farrar, G. R. \& Piran, T., 2000, arXiv:astro-ph/0010370 Farrar, G. R.\& Gruzinov, A. 2009, ApJ, 693, 329

Finke, J. D.; Razzaque, S.; Dermer, C. D., 2010, ApJ, 712, 238

Gabici, S. \& Aharonian, F. A., 2005, Phys. Rev. Lett., 95, 251102

Guetta, D.; Piran, T.; Waxman, E., 2005, ApJ, 619, 412

Hasinger, G.; Miyaji, T.; Schmidt, M., 2005, A\&A, 441, 417

Inoue, S.; Aharonian, F. A.; Sugiyama, N., 2005, ApJ, 628, L9

Kalashev, O. E.; Semikoz, D. V.; Sigl, G., 2009, Phys. Rev. D, 79, 063005

Karakula, S., \& Tkaczyk, W. 1993, Astroparticle Physics, 1, 229

Kotera, K., Allard, D. \& Lemoine, M., 2011, A\&A, arXiv:1011.0575

Kistler, M. D.; Yüksel, H.; Beacom, J. F.; Stanek, K. Z., ApJ, 2008, 673, L119

Langer, N.; Norman, C. A., 2006, ApJ, 638, L63

Le, T. \& Dermer, C. D., 2007, ApJ, 661, 394

Lemoine, M., 2005, Phys. Rev. D, 71, 083007

Murase, K., Ioka, K., Nagataki, S., \& Nakamura, T. 2008, Phys. Rev. D, 78, 023005

Mücke, A., Engel, R., Rachen, J. P., Protheroe, R. J., \& Stanev, T. 2000, Comput. Phys. Commun., 124, 290

Neronov, A., \& Semikoz, D. V., 2011, axXiv:1103.3484

Puget, J. L.; Stecker, F. W.; Bredekamp, J. H., 1976, ApJ, 205, 638
Rieger, F. M.; Aharonian, F. A., 2009, A\&A, 506, L41

Ryu, D., Kang, H. \& Biermann, P. L., 1998, A\&A, 335, 19

Stanek, K. Z. et al. 2006, Acta Astronomica, 56, 333

Stecker, F. W.; Salamon, M. H., 1999, ApJ, 512, 521

Strong, A. W., Wdowczyk, J., \& Wolfendale, A. W. 1973, Nature, 241,109

Vietri, M. 1995, ApJ, 453, 883

Wanderman, D. \& Piran, T., 2010, Phys. Rev. D, 406, 1944

Wang X. Y., Razzaque, S., Mészáros, P. \& Dai, Z. G., 2007, Phy. Rev. D, 6, 083009

Waxman, E., 1995, Phys. Rev. Lett., 75, 386

Waxman, E., Bahcall, J., 1999, Phys. Rev. D, 59, 023002

Wick, S. D., Dermer, C. D., \& Atoyan, A. 2004, Astroparticle Physics, 21, 125

Wdowczyk, J.; Tkaczyk, W.; Wolfendale, A. W., 1972, Journal of Physics A: Mathematical and General, 5, 1419

Yüksel, H. \& Kistler, M. D. 2007, Phys. Rev. D, 75, 083004

Yüksel, H.; Kistler, M. D.; Beacom, J. F.; Hopkins, A. M., 2008, ApJ, 638, L5 


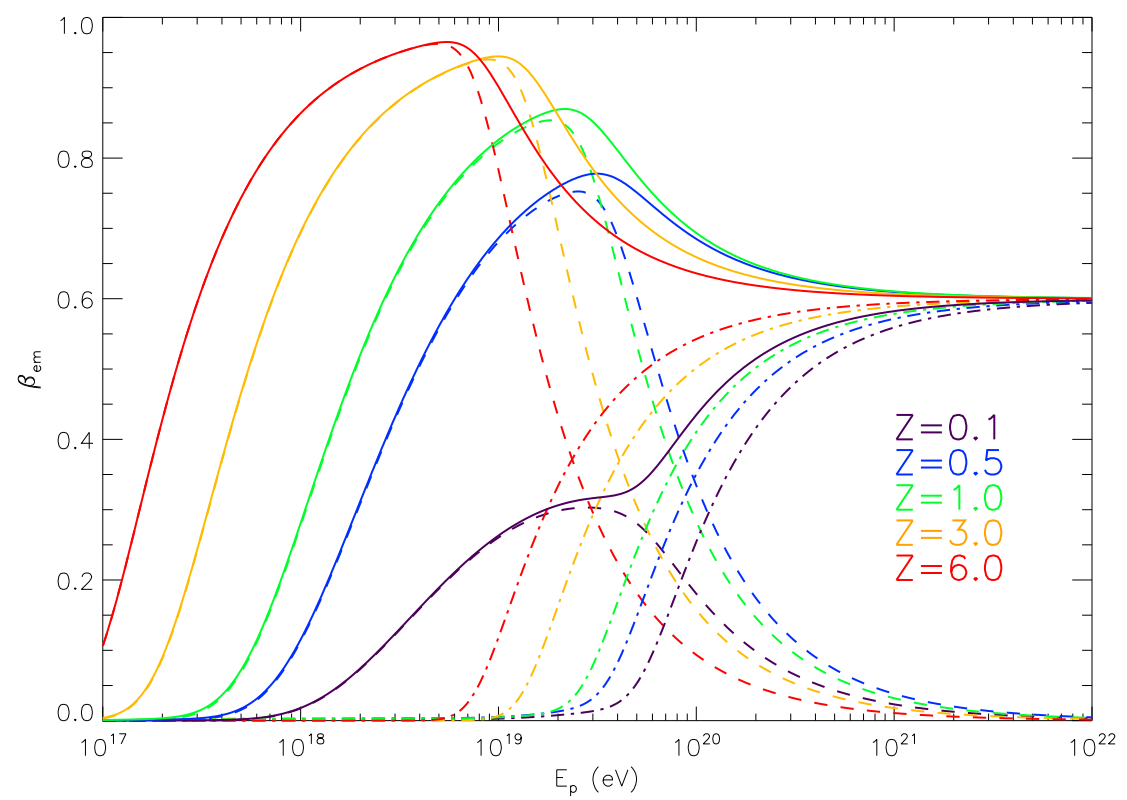

FIG. 1. - The fraction energy loss of UHE protons into the electro-magnetic component for protons generated at different redshifts. The dashed lines and dash dotted lines represent the fractions of energy loss through the photopair channel and photopion channel respectively, while the solid lines represent the sum of them. 

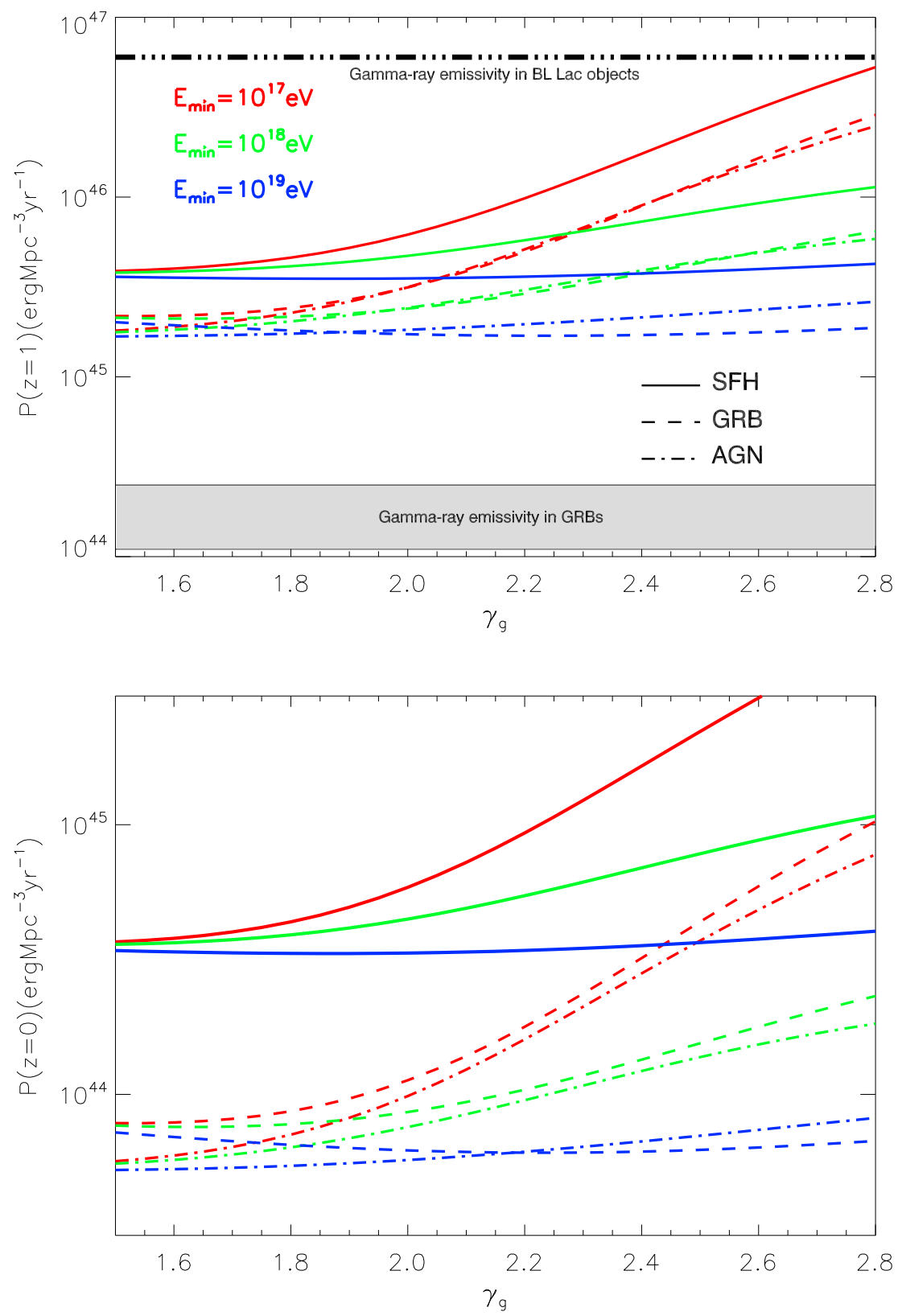

FIG. 2.- Upper limits on the UHECR emissivity as a function of the spectral index of the cosmic ray proton spectrum for different source density evolution scenarios. The upper panel shows the upper limits on the UHECR emissivity at $z=1$, and the bottom panel shows the upper limits on the UHECR emissivity at the present time $(z \ll 1)$ if the source density evolution follows $S(z)$ up to the present time. The maximum energy is fixed at $E_{p, \max }=10^{21} \mathrm{eV}$, while three values are assumed for the minimum energy. In the upper panel, the gamma-ray emissivity of candidate UHECR sources (GRBs and BL Lac objects) at $z=1$ are plotted for comparison. 

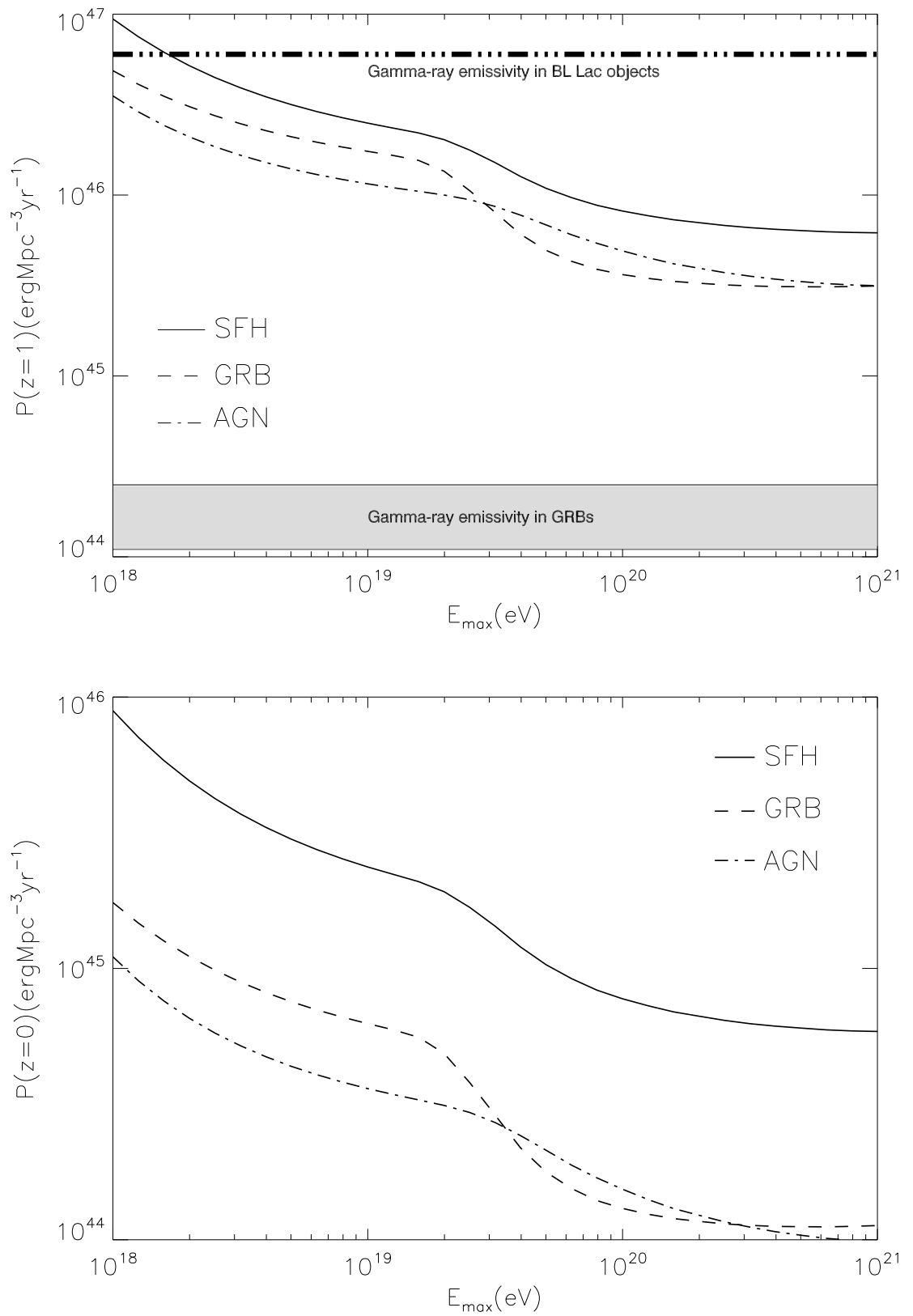

FIG. 3.- Upper limits on the UHECR emissivity as a function of the maximum energy of the cosmic ray spectrum for different source density evolution scenarios. The spectral index of the the cosmic ray spectrum is fixed at $\gamma_{g}=2$ and $E_{p, \min }=10^{17} \mathrm{eV}$. 


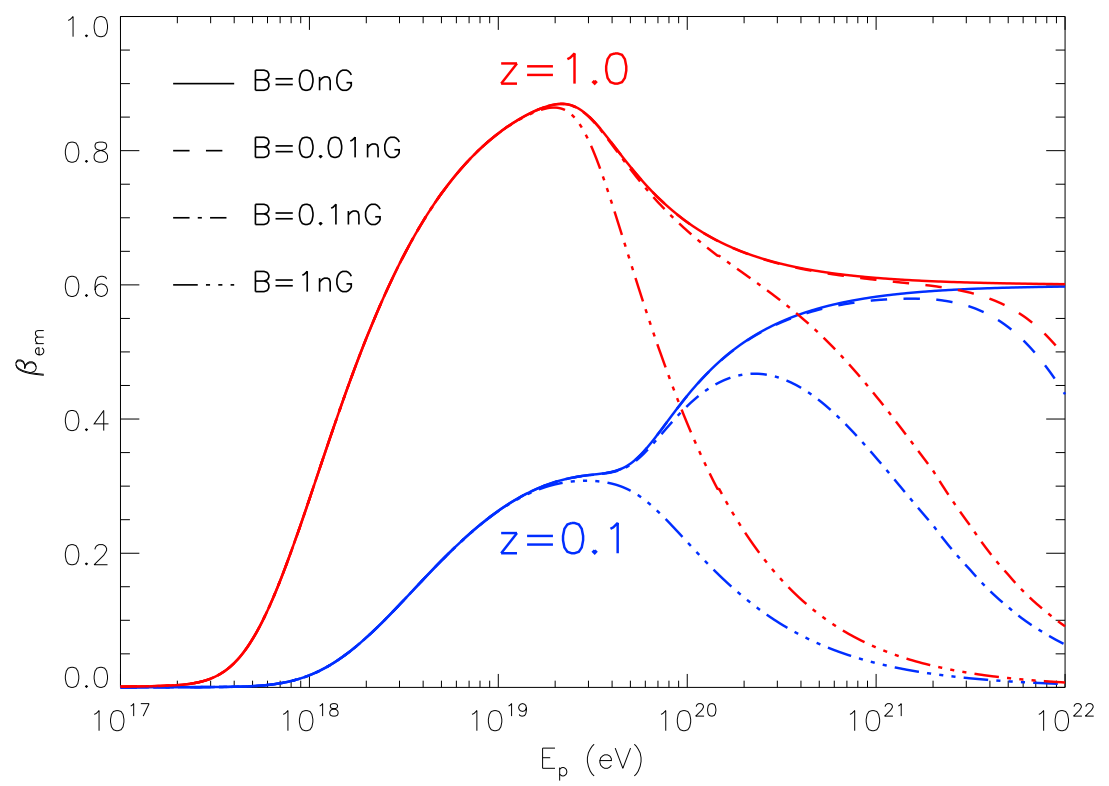

FIG. 4.- The fraction energy loss of UHE protons into the electro-magnetic component for protons generated at two different redshifts in the presence of intergalactic magnetic fields. Different lines correspond to different strength of the intergalactic magnetic fields.

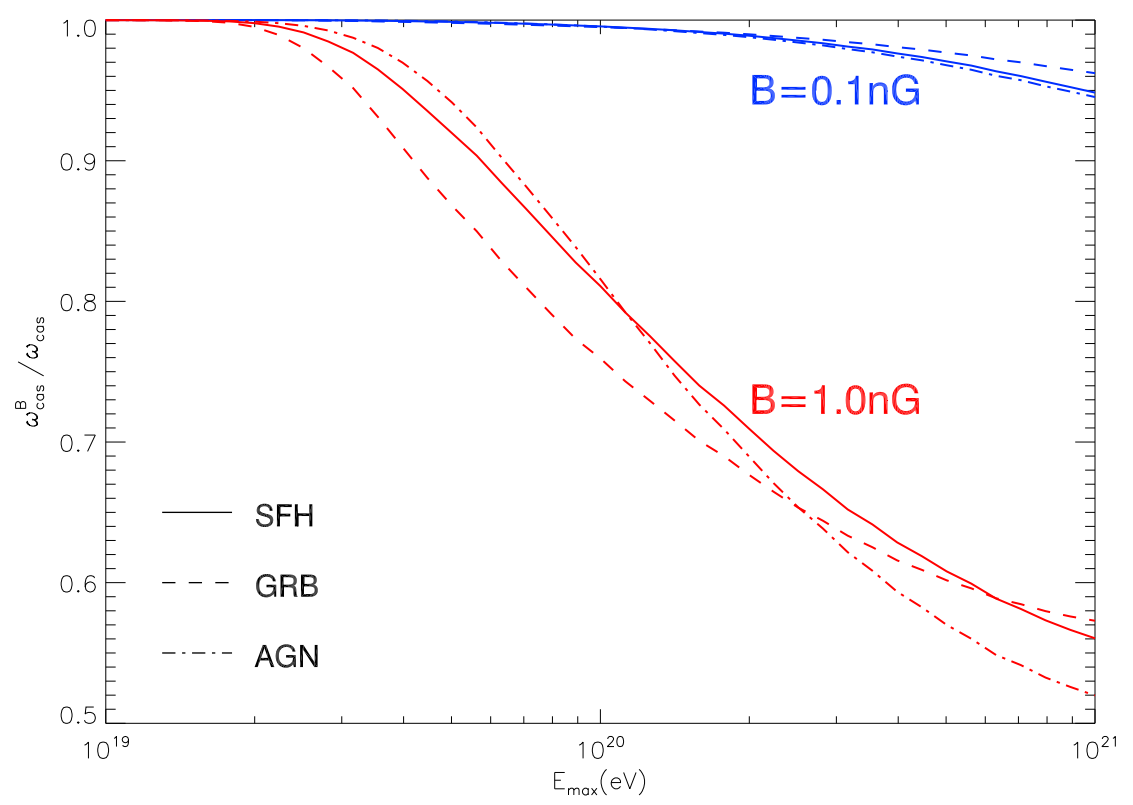

FIG. 5.- The ratios of the cascade energy densities in the presence and in the absence of intergalactic magnetic fields. The blue and red lines correspond to the ratios for intergalactic magnetic fields of $0.1 \mathrm{nG}$ and $1 \mathrm{nG}$, respectively. $E_{p, \min }=10^{17} \mathrm{eV}$ and $\gamma_{g}=2$ are used in the calculation. 


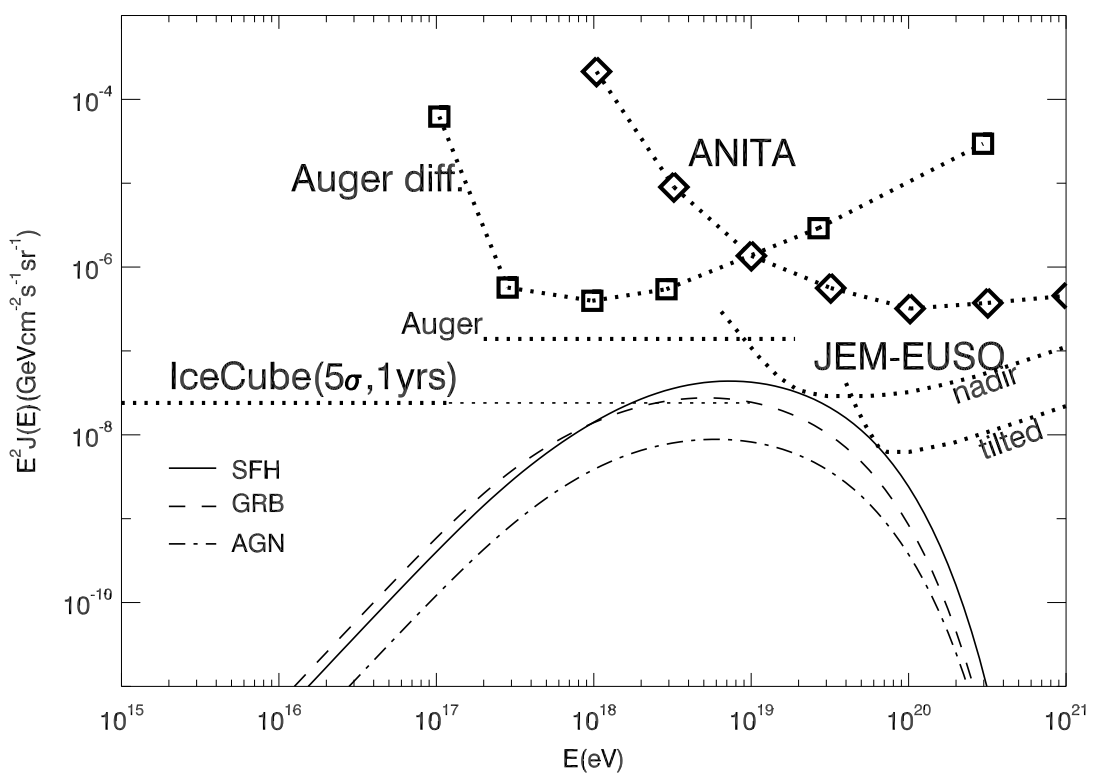

FIG. 6.- Upper limits on the cosmogenic neutrino flux $\left(\nu_{\mu}+\bar{\nu}_{\mu}\right)$, derived from the upper limits of the UHECR emissivity in the universe, for different source density evolution scenarios. $E_{p, \min }=10^{17} \mathrm{eV}, E_{p, \max }=10^{21} \mathrm{eV}$ and $\gamma_{g}=2$ are used in the calculation. 


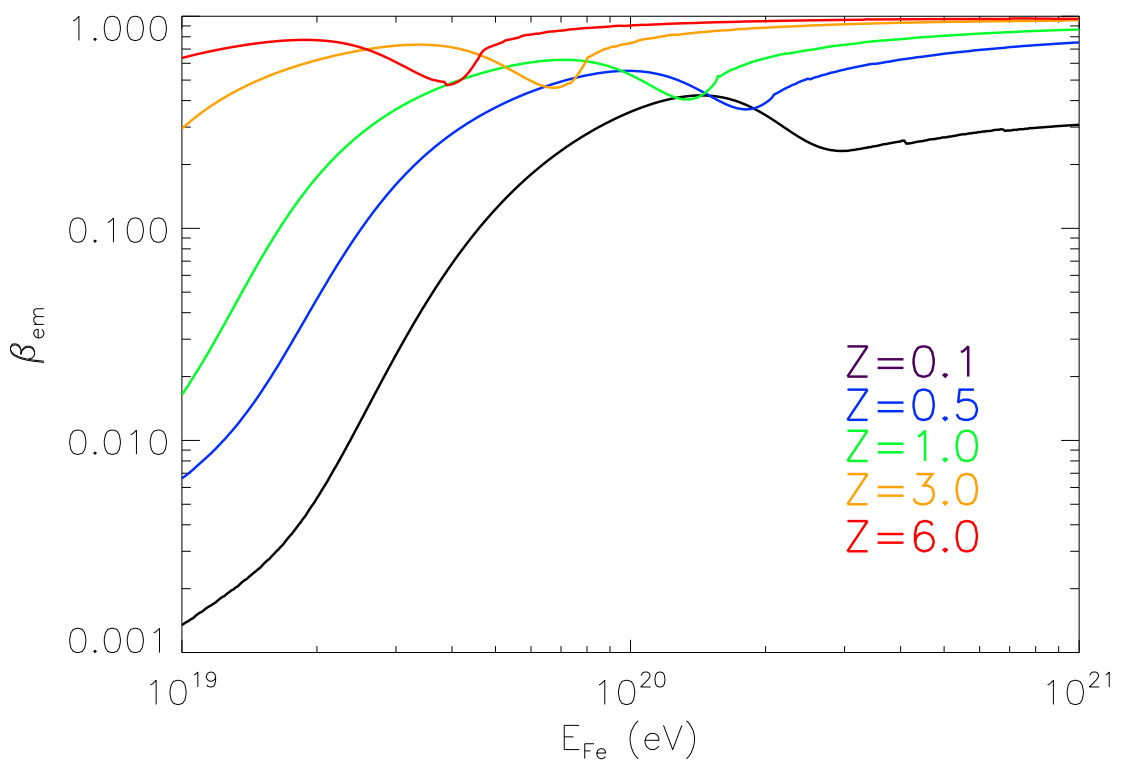

FIG. 7.- The fraction energy loss of UHE iron nuclei into the electro-magnetic component for iron nuclei generated at different redshifts. 

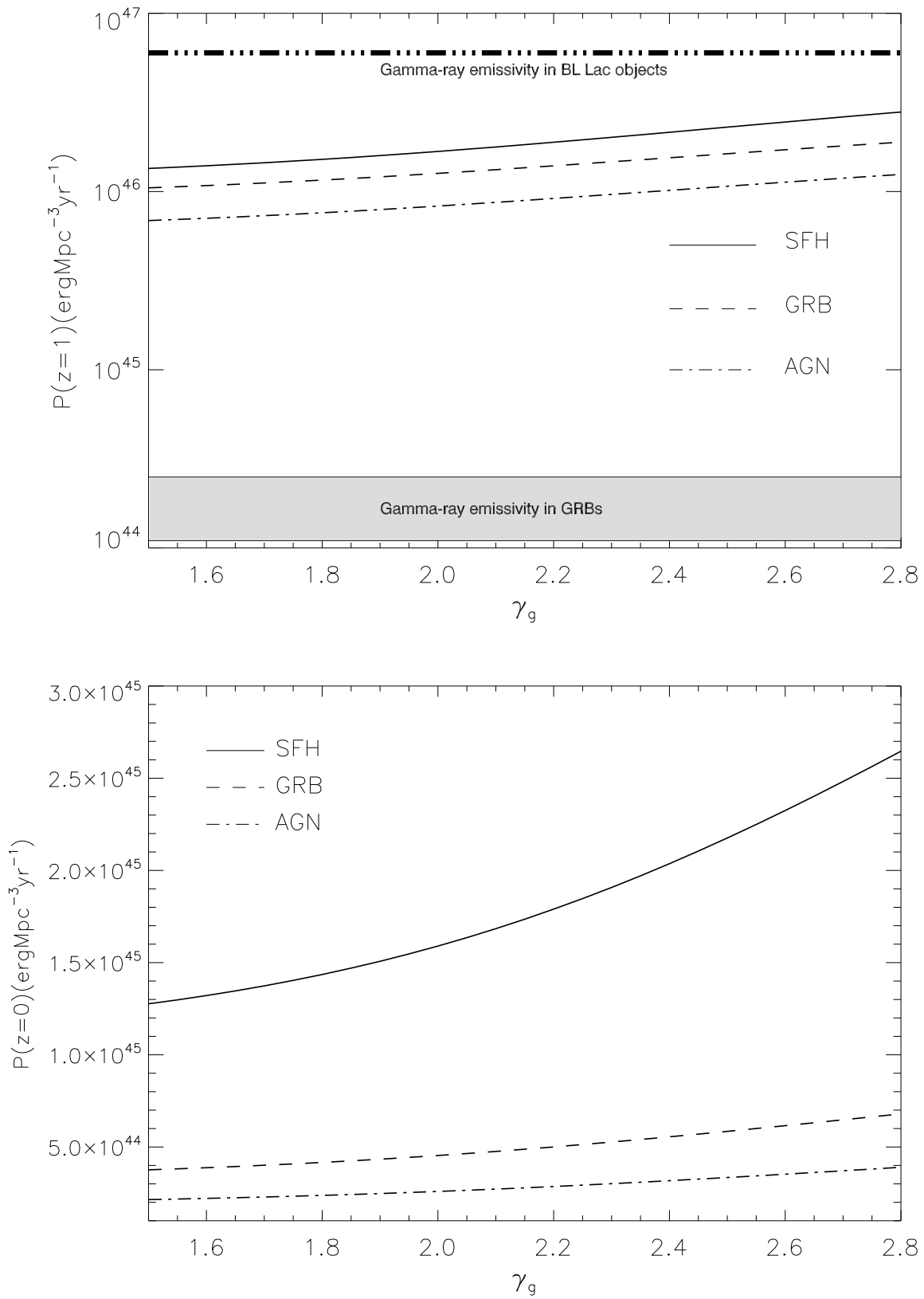

FIG. 8.- Upper limits on the UHECR emissivity as a function of the spectral index for a pure iron nuclei composition of UHECRs in the energy range from $E_{\min }=10^{19} \mathrm{eV}$ to $E_{\max }=10^{21} \mathrm{eV}$. 